\title{
Criminologie
}

\section{Le contrôle automatisé de la vitesse en Australie : quelques enseignements pour mener une politique de dissuasion efficace}

\section{Laurent Carnis}

Volume 41, numéro 2, automne-hiver 2008

Les organisations dites sectes, les lois et la société

URI : https://id.erudit.org/iderudit/019440ar

DOI : https://doi.org/10.7202/019440ar

Aller au sommaire du numéro

Éditeur(s)

Les Presses de l'Université de Montréal

ISSN

0316-0041 (imprimé)

1492-1367 (numérique)

Découvrir la revue

Citer cet article

Carnis, L. (2008). Le contrôle automatisé de la vitesse en Australie : quelques enseignements pour mener une politique de dissuasion efficace. Criminologie, 41(2), 269-290. https://doi.org/10.7202/019440ar

\section{Résumé de l'article}

Le contrôle automatisé de la vitesse (CAV) constitue une modalité d'intervention des autorités pour réduire le nombre de victimes de la route. Cette technique de détection permet d'accroître l'intensité des contrôles, d'augmenter la sanction potentielle des contrevenants et de rendre possible une procédure judiciaire plus rapide. Ce type de dispositif a été introduit pour la première fois en Australie au milieu des années 1980 dans l'État de Victoria. Depuis, cette technique s'est généralisée à l'ensemble des États. Les dispositifs en fonctionnement se révèlent fort divers et s'appuient sur des stratégies spécifiques. Néanmoins, ces expériences convergent vers une même conclusion : la diminution significative des accidents de la circulation et le nombre de victimes. Ils s'avèrent donc particulièrement efficaces. Par ailleurs, ces différents programmes de contrôle s’appuient sur des architectures organisationnelles et institutionnelles différentes, illustrant par la même une certaine souplesse quant à leur implantation dans des environnements particuliers. Toutefois, ils reposent tous sur les principes simples des « 4C » (contrôle, coopération, communication et continuité), nécessitant l'intégration des acteurs clés. 


\title{
Le contrôle automatisé de la vitesse en Australie : quelques enseignements pour mener une politique de dissuasion efficace
}

\author{
Laurent Carnis \\ Chargé de recherche \\ Institut National de Recherche sur les Transports et leur Sécurité (INRETS) \\ laurent.carnis@inrets.fr
}

\begin{abstract}
RÉSUMÉ - Le contrôle automatisé de la vitesse (CAV) constitue une modalité d'intervention des autorités pour réduire le nombre de victimes de la route. Cette technique de détection permet d'accroître l'intensité des contrôles, d'augmenter la sanction potentielle des contrevenants et de rendre possible une procédure judiciaire plus rapide. Ce type de dispositif a été introduit pour la première fois en Australie au milieu des années 1980 dans l'État de Victoria. Depuis, cette technique s'est généralisée à l'ensemble des États. Les dispositifs en fonctionnement se révèlent fort divers et s'appuient sur des stratégies spécifiques. Néanmoins, ces expériences convergent vers une même conclusion: la diminution significative des accidents de la circulation et le nombre de victimes. Ils s'avèrent donc particulièrement efficaces. Par ailleurs, ces différents programmes de contrôle s'appuient sur des architectures organisationnelles et institutionnelles différentes, illustrant par la même une certaine souplesse quant à leur implantation dans des environnements particuliers. Toutefois, ils reposent tous sur les principes simples des «4C» (contrôle, coopération, communication et continuité), nécessitant l'intégration des acteurs clés.
\end{abstract}

\section{Introduction}

La réduction de l'insécurité routière constitue de nos jours un objectif clairement affiché pour la plupart des autorités publiques les obligeant à définir des programmes d'intervention pluriannuels et des orientations stratégiques. La prise en compte de cette dimension au sein des politiques publiques fait suite à l'utilisation de masse des véhicules à moteur qui s'accompagne d'une augmentation de l'accidentalité. Cette situation 
nécessite une réponse de la part des autorités, puisque le problème ne s'avère aucunement passager; de surcroît, il concerne les voies publiques et ne peut se résoudre de lui-même.

Depuis plus de 20 ans, la mise en œuvre de dispositifs de contrôle automatisé de la vitesse (CAV) s'est généralisée en Australie, prenant des formes relativement élaborées pour devenir une technique de détection et de sanction incontournable. Elle permet à la fois une intensification des contrôles des contrevenants potentiels, une sanction potentielle accrue et un traitement pénal de l'infraction plus rapide. Le CAV propose ainsi une réponse adaptée en permettant le traitement de masse d'un phénomène de masse, l'infraction au Code de la route.

Au-delà des répercussions sur l'accidentalité, le respect des limitations de vitesse et sur les vitesses de circulation, le CAV constitue une modernisation évidente de l'intervention publique dans le domaine de la sécurité routière. La lutte contre la vitesse excessive constitue une cible sur laquelle les autorités agissent et représente un objet exploratoire préfigurant l'intervention publique des prochaines années en sécurité routière'.

L'étude du cas australien, ou plus précisément des cas australiens, puisqu'il existe un éventail d'expériences diverses, permet de constater les effets que l'on peut anticiper d'un tel dispositif. L'Australie, État fédéral, n'a pas empêché l'élaboration de réponses locales prenant en compte les spécificités de chaque État. L'étude de ces dispositifs met en évidence une grande diversité tant dans les objectifs suivis que dans les modalités opérationnelles adoptées, tout en présentant des résultats convergents en termes de réduction de l'insécurité routière (première section de ce texte). Cette diversité se traduit également dans la définition de structures organisationnelles et institutionnelles variées, qui reposent pourtant sur des principes d'intervention communs (deuxième section). En cela, les expériences australiennes fournissent des enseignements précieux pour des autorités soucieuses d'élaborer et d'implanter un dispositif de contrôle des vitesses.

1. Les autorités disposent ou disposeront à l'avenir de dispositifs automatisés concernant le respect des feux rouges, le pesage des véhicules en marche, le contrôle du paiement des voies à péage et le contrôle des interdistances entre véhicules. D'autres dispositifs sont à l'étude concernant le passage des voies ferrées, la circulation dans les tunnels et la compatibilité des dispositifs de contrôle manuels vers un mode automatisé. 


\section{Les dispositifs automatisés australiens de CAV: entre diversité des programmes et enseignements convergents}

La diversité des systèmes australiens se distingue tant par la pluralité des environnements au sein desquels ils ont été implantés que par une temporalité désynchronisée, une taille et une composition de dispositifs différents. Toutefois, la diversité institutionnelle des dispositifs de CAV n'empêche pas l'émergence d'enseignements communs: un processus de dissuasion performant qui doit toutefois composer avec certaines limites.

\section{Une grande diversité de systèmes}

La diversité des environnements implique des dispositifs différenciés

Les huit provinces australiennes présentent une forte hétérogénéité (Harwood et al., 2001 : 15). En effet, le territoire de la capitale fédérale (ACT) représente seulement $2400 \mathrm{~km}^{2}$, tandis que l'Australie-Occidentale dispose d'un territoire relativement vaste de près de 2,5 millions de $\mathrm{km}^{2}$, soit un rapport de 1 à 100 entre la juridiction la plus petite et la plus vaste. De manière similaire, le rapport entre le territoire le plus peuplé (Nouvelle-Galles du Sud) et le moins pourvu (Territoires du Nord) est de 35. Cette hétérogénéité s'exprime également par des densités géographiques fort variées avec des provinces peu peuplées avec moins d'un habitant au $\mathrm{km}^{2}$ telles que les Territoires du Nord ou encore l'Australie-Occidentale, alors que l'État de Victoria présente une densité de plus de 20 habitants au $\mathrm{km}^{2}$. Canberra dispose de la densité la plus élevée. Sans aller plus en avant dans les détails, on doit retenir de cette diversité environnementale l'existence de réseaux de taille différente, des populations plus ou moins conséquentes à surveiller et des zones de contrôle aux caractéristiques urbaines variables. Ces différentes dimensions exigent ainsi d'adapter et de préciser en conséquence le dispositif de contrôle (Opp et al., 1972). En effet, plus la population à surveiller est importante, plus la taille du dispositif devra augmenter plus que proportionnellement ${ }^{2}$. De même, pour un réseau

2. On renvoie ici le lecteur aux conséquences productives des rendements décroissants. Ce point est analysé plus loin, dans la section relative à la convergence des enseignements. 
de longueur plus importante, les efforts de surveillance consacrés devront être plus importants pour maintenir la même probabilité de détection. Par ailleurs, une circulation plus urbaine signifie une concentration des déplacements nécessitant une automatisation des contrôles par l'intermédiaire, entre autres, de dispositifs fixes (Cameron et al., 2003a). Ainsi, le Queensland a opté pour un dispositif de contrôle orienté vers les zones rurales, tandis que l'Australie-Occidentale et la Nouvelle-Galles du Sud ont privilégié une intervention urbaine. Enfin, l'État de Victoria a adopté une stratégie mixte, déployant ses équipements à la fois sur les réseaux rural et urbain.

Les contraintes sociétales influent également sur le fonctionnement du dispositif

Toutefois, même si les conditions environnementales façonnent le dispositif du contrôle automatisé, elles ne permettent pas d'expliquer les spécificités des programmes de contrôle mis en œuvre par chaque État. En effet, les contraintes sociétales ${ }^{3}$ influent également sur leur mise en œuvre. Ainsi, on constate tout d'abord une implantation désynchronisée des différents dispositifs de contrôle (voir tableau 1).

TABLEAU 1

Date d'introduction et type de dispositif des systèmes de contrôle automatisé de la vitesse pour les différents États australiens

\begin{tabular}{|l|l|l|l|l|l|l|l|l|}
\hline & Victoria & $\begin{array}{l}\text { Nouvelle-Galles } \\
\text { du Sud }\end{array}$ & $\begin{array}{l}\text { Australie } \\
\text { du Sud }\end{array}$ & Tasmanie & Queensland & $\begin{array}{l}\text { Australie- } \\
\text { Occidentale }\end{array}$ & $\begin{array}{l}\text { Territoires } \\
\text { du Nord }\end{array}$ & ACT \\
\hline $\begin{array}{l}\text { Date } \\
\text { d'intro- } \\
\text { duction }\end{array}$ & $\begin{array}{l}(1985- \\
1986) \\
1989\end{array}$ & $\begin{array}{l}1991 \\
\text { (mobile) } \\
1999 \text { (fixe) }\end{array}$ & $\begin{array}{l}1990- \\
1991\end{array}$ & 1993 & 1997 & $\begin{array}{l}(1988) \\
1998\end{array}$ & 1998 & 1999 \\
$\begin{array}{l}\text { Type de } \\
\text { dispositif }\end{array}$ & Mixte & Mixte & Mobile & Mixte & Mobile & $\begin{array}{l}\text { Mobile } \\
\text { (Mixte en } \\
\text { projet) }\end{array}$ & Mobile & Fixe \\
\hline
\end{tabular}

Ainsi, on peut distinguer trois grandes vagues d'implantation des systèmes de contrôle automatisé de la vitesse en Australie. Une première phase introductive commence au début de la décennie 1980. L'État de Victoria apparaît comme le précurseur avec une première expérimenta-

3. On désigne par contraintes sociétales à la fois des contraintes d'ordre économique (base fiscale plus ou moins importante, dynamisme économique, etc.), d'ordre social (acceptabilité des dispositifs, rapport à la voiture, à la vitesse) et d'ordre politique (organisation politique de l'État, position consensuelle des partis sur le contrôle des vitesses). 
tion menée en 1986, même si ce n'est qu'à partir de 1989 que débute réellement le programme de contrôle et qu'il s'intensifie à partir de 1990-1991 (Bourne et al., 1993). En 1988, un embryon de système est également mis en œuvre en Australie-Occidentale avec le déploiement de quelques appareils.

Une deuxième vague d'implantations des CAV a conduit au début des années 1990 à diffuser cette modalité d'intervention. Le dispositif de contrôle des vitesses à Victoria s'intensifie. De nouveaux systèmes apparaissent dans les États de Nouvelle-Galles, d'Australie du Sud et en Tasmanie.

Le troisième mouvement prend forme à la fin des années 1990. Il s'agit d'une phase de généralisation de la technique de contrôle à l'ensemble des États. La Nouvelle-Galles du Sud introduit des dispositifs fixes renforçant ses appareils mobiles, l'Australie-Occidentale intensifie son programme de contrôle, tandis que le Queensland, les Territoires du Nord et Canberra mettent en œuvre leur propre programme. Cette typologie chronologique permet certes de distinguer des mouvements sans toutefois apporter des explications à une diffusion qui s'étend tout de même sur environ 10 années, et ce, malgré la proximité géographique.

L'hétérogénéité des dispositifs peut également être observée en comparant la taille des programmes de contrôle. Différents indicateurs peuvent être mobilisés, comme le nombre d'appareils automatisés ou de sites de contrôle qui peuvent être rapportés à la superficie du territoire, à la taille du réseau, au kilométrage effectué ou encore à la taille de la population. (Harwood et al., 2001: 155). Ainsi, l'État de Victoria, qui présente un nombre d'appareils relativement élevé dispose d'une intensité de contrôle beaucoup plus faible lorsque la taille de la population est prise en considération. En Nouvelle-Galles du Sud et surtout au Queensland, le nombre d'appareils s'avère plus faible (de moitié inférieur à celui de Victoria), mais l'intensité de surveillance est beaucoup plus importante.

Par ailleurs, les différents États ont maintenu voire développé les différents moyens traditionnels de contrôle (appareils laser, radars traditionnels, etc.). Ainsi, la Nouvelle-Galles du Sud était équipée en 1998 de plus de 600 autres appareils de contrôle, tandis que le Queensland ne disposait que de 300 appareils manuels pour un nombre quasi identique d'appareils automatisés.

Deux autres éléments permettent d'examiner ces cheminements institutionnels distincts. Premièrement, la sévérité de la sanction pour un 
excès de vitesse diffère substantiellement d'un État à un autre. Ainsi, en 1998, le minimum des amendes variait du simple au double selon les États, tandis que le maximum s'échelonnait selon un rapport de 1 à 5 . De même, certains États prévoient une sanction avec le retrait de points du permis de conduire, ce qui n'est toutefois pas le cas des Territoires du Nord. En fait, la structure des sanctions dans cet État suggère une relative clémence à l'égard des contrevenants à la limitation de vitesse. Par ailleurs, le cas de la Nouvelle-Galles du Sud apparâtt particulièrement intéressant dans la mesure où il se distingue clairement des pratiques des autres États par une extrême sévérité à l'égard des contrevenants. L'État a d'ailleurs récemment introduit un dispositif spécial qui prévoit de multiplier par deux le retrait de points du permis de conduire pour les excès de vitesse commis pendant les périodes de fêtes et de vacances. Le cas du Queensland suscite aussi un certain intérêt dans la mesure où le renforcement du contrôle des vitesses s'est conjointement accompagné d'un renforcement de la sévérité des sanctions. Un réel changement institutionnel s'est opéré dans cet État, alors que les autres États ne démontrent pas une telle évolution.

TABLEAU 2

Sanctions encourues pour un excès de vitesse

\begin{tabular}{|c|c|c|c|c|c|c|c|c|}
\hline & Victoria & $\begin{array}{l}\text { Nouvelle- } \\
\text { Galles du } \\
\text { Sud }\end{array}$ & $\begin{array}{l}\text { Australie } \\
\text { du Sud }\end{array}$ & Tasmanie & Queensland & $\begin{array}{l}\text { Australie- } \\
\text { Occidentale }\end{array}$ & $\begin{array}{l}\text { Territoires } \\
\text { du Nord }\end{array}$ & $A C T$ \\
\hline Amende & $\begin{array}{l}\text { De } 105 \text { à } \\
360 \$\end{array}$ & $\begin{array}{l}\text { De } 112 \text { à } \\
1380 \$\end{array}$ & $\begin{array}{l}\text { De } 116 \text { à } \\
298 \$\end{array}$ & $\begin{array}{l}\text { De } 50 \text { à } \\
170 \$\end{array}$ & $\begin{array}{l}\text { De } 80 \text { à } \\
250 \$\end{array}$ & $\begin{array}{l}\text { De } 50 \text { à } \\
350 \$\end{array}$ & $\begin{array}{l}\text { De } 50 \text { à } \\
150 \$\end{array}$ & $\begin{array}{l}\text { De } 112 \text { à } \\
690 \$\end{array}$ \\
\hline $\begin{array}{l}\text { Points } \\
\text { retirés } \\
1998\end{array}$ & De 1 à 6 & De 1 à 6 & De 1 à 6 & De 1 à 6 & De 1 à 6 & De 0 à 6 & Aucun & De 1 à 6 \\
\hline Amende & $\begin{array}{l}\text { De } 210 \text { à } \\
451 \$\end{array}$ & $\begin{array}{l}\text { De } 123 \text { à } \\
1515 \$\end{array}$ & $\begin{array}{l}\text { De } 100 \text { à } \\
350 \$\end{array}$ & $\begin{array}{l}\text { De } 50 \text { à } \\
400 \$\end{array}$ & $\begin{array}{l}\text { De } 100 \text { à } \\
700 \$\end{array}$ & $\begin{array}{l}\text { De } 100 \text { à } \\
350 \$\end{array}$ & $\begin{array}{l}\text { De } 100 \text { à } \\
350 \$\end{array}$ & $\begin{array}{l}\text { De } 133 \text { à } \\
1637 \$\end{array}$ \\
\hline $\begin{array}{l}\text { Points } \\
\text { retirés } \\
2006\end{array}$ & De 1 à 8 & De 2 à 6 & De 1 à 6 & De 1 à 6 & De 1 à 8 & De 1 à 6 & Aucun & De 1 à 6 \\
\hline
\end{tabular}

Le choix des modalités de contrôle diffère également d'un État à un autre. Ainsi, l'État de Victoria dispose d'un système essentiellement composé de dispositifs mobiles signalés. Plus récemment, les autorités ont introduit des dispositifs fixes non signalés: les panneaux de signalisation avertissant du lieu de contrôle ayant été retirés. En NouvelleGalles du Sud, les dispositifs mobiles et fixes sont signalés. Cependant, 
les dispositifs mobiles sont déployés pour résoudre les problèmes de points noirs (zones géographiques se caractérisant par une recrudescence d'accidents corporels) et ont largement précédé le déploiement des dispositifs fixes. Enfin, le Queensland utilise des dispositifs mobiles dont la zone de contrôle est signalée et non le lieu en tant que tel ${ }^{4}$. Le choix des lieux de contrôle repose sur un processus de détermination aléatoire, ce qui créé ainsi une dissuasion accrue pour le conducteur placé dans une situation d'incertitude plus importante. On comprend ici que l'éventail des stratégies disponibles et leur évolution même au sein de chaque dispositif (évolution de dispositifs signalés vers des appareils cachés, évolution s'appuyant uniquement sur des dispositifs mobiles vers des combinaisons d'appareils fixes et mobiles, etc.) ne peut être expliquée par le seul aspect environnemental. Certes l'environnement influe sur les modalités de contrôle et les choix effectués par les autorités dans la mesure où ils contribuent à déterminer les enjeux et les contraintes d'intervention. Néanmoins, il ne permet pas d'expliquer la grande diversité des expériences australiennes. En conséquence, les dynamiques institutionnelles propres à chaque État doivent être prises en compte pour être en mesure de comprendre ces différences de fonctionnement substantielles. Seuls des cheminements institutionnels particuliers peuvent expliquer à la fois les différences de temporalité, les choix qui ont présidé à mettre en œuvre des systèmes plus ou moins prégnants et des sanctions plus ou moins sévères obéissant à des stratégies variées. En fait, comme l'a souligné Becker (1968) la mise en œuvre d'une politique publique de lutte contre une forme de criminalité, quelle qu'elle soit, révèle par l'importance des moyens mis en œuvre le niveau de criminalité qui restera impuni et qui s'avère donc socialement acceptable.

\section{La convergence des enseignements}

Une technique de dissuasion performante

Du fait de programmes aux caractéristiques distinctes et aux dimensions différentes, la démarche qui consisterait à synthétiser l'ensemble des résultats à partir d'une même échelle nous paraît risquée, voire erronée,

4. En fait, le lieu de contrôle est signalé au conducteur après son passage et non avant. De plus, les véhicules sont facilement identifiables. 
du point de vue méthodologique 5 . Néanmoins, les travaux d'évaluation réalisés convergent pour affirmer l'efficacité des CAV.

$\mathrm{Au}$ Queensland, la vitesse moyenne a chuté de $6 \mathrm{~km} / \mathrm{h}$ environ. La diminution $\mathrm{du} 85^{\mathrm{e}}$ centile s'avère du même ordre avec une réduction de $7 \mathrm{~km} / \mathrm{h}$. Le pourcentage de conducteurs ne respectant pas les limites de vitesse est passé de 53 à 16\% (Champness et al., 2005). À Canberra, le déploiement du dispositif a permis de réduire de $26 \%$ le pourcentage de conducteurs en situation d'excès de vitesse et de $63 \%$ pour les excès de vitesse de plus de $10 \mathrm{~km} / \mathrm{h}$ (Anderson et al., 2001). Des travaux récents menés sur la Nouvelle-Galles du Sud mettent en évidence une réduction de $6 \mathrm{~km} / \mathrm{h}$ de la vitesse moyenne de $70 \%$ du nombre d'excès de vitesse. Cette réduction se situe autour de $85 \%$ environ pour les excès de vitesse supérieurs à 10, 30 et $40 \mathrm{~km} / \mathrm{h}$ (ARRB, 2005). Dans l'État de Victoria, le doublement des heures de contrôle (de 2000 heures à environ 4000 heures mensuelles) a permis de réduire le taux d'infraction à la limite de vitesse à un niveau proche de $2 \%$, c'est-à-dire une élimination presque totale des infractions aux lieux de contrôle (voir figure 1).

L'évaluation des effets relatifs aux dispositifs de contrôle fixes met en évidence une diminution de la vitesse moyenne de $2,5 \mathrm{~km} / \mathrm{h}$ et une réduction de la proportion des conducteurs en situation d'excès de vitesse de plus de $60 \%$ pour les limitations de vitesse les plus faibles et de plus de $70 \%$ pour les plus élevées (Cameron et al., 2003b).

\section{Des performances notables sur l'insécurité routière}

La réduction des vitesses de circulation entrâne également une diminution de l'insécurité routière. L'étude des différents dispositifs de contrôle automatisé souligne la bonne transmission des effets entre l'activité de surveillance et l'impact sur le nombre de victimes et d'accidents. L'augmentation des contrôles conduit à une baisse de la vitesse de circulation et à une réduction du risque routier. Ce résultat tranche quelque peu avec les effets associés aux contrôles traditionnels, pour lesquels la transmission entre l'augmentation de la surveillance policière et la réduction des vitesses n'était pas aussi évidente et faisait l'objet de débats. L'impact sur le risque routier était l'objet également de discus-

5. Avant même de se questionner sur la possibilité de synthétiser les résultats, il conviendrait de s'interroger sur la méthodologie utilisée pour parvenir à produire ces résultats. 
FIG URE 1

Évolution du taux d'infraction à la limite de vitesse et des heures de surveillance entre juin 1990 et mars 1997 dans l'État de Victoria

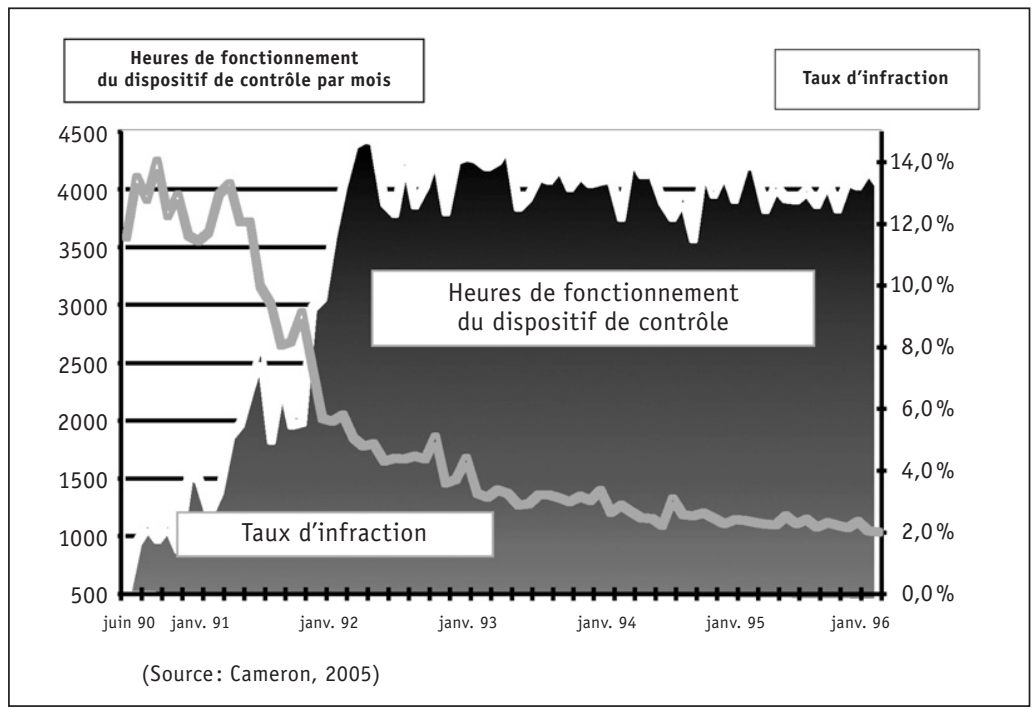

sions scientifiques. En effet, les contrôles manuels laissent place, entre autres, à l'arbitraire policier (Carnis, 2001a), qui constitue autant de pertes $^{6}$ d'efficacité du dispositif de contrôle (Carnis, 2001b). En somme, les CAV permettent une plus grande efficacité dans l'activité de contrôle grâce à une capacité accrue de détection et de sanction (intensification des contrôles et procédures de poursuite de masse). Sur ce point, il existe également une réelle convergence des résultats.

Ainsi au Queensland, la réduction du nombre de personnes tuées est estimée à environ $16 \%$ et la diminution des accidents matériels à proximité des lieux de contrôle à $20 \%$ environ (Newstead et al., 2003 : 13). L'importance de ces estimations varie cependant selon le critère spatial d'évaluation. De même, une réduction de $36 \%$ des accidents avec des personnes blessées est associée à la mise en œuvre du CAV dans la ville de Canberra. Cet effet fait suite à une réduction substantielle de la proportion des conducteurs en situation d'excès de vitesse

6. L'automatisation des contrôles permet ainsi d'homogénéiser les pratiques en matière de seuils de tolérance (seuils de vitesse à partir desquels le conducteur devient un contrevenant) et de réduire les possibilités d'indulgence (abandon arbitraire des poursuites). 
(Anderson et al., 2001). En Nouvelle-Galles du Sud, les accidents matériels et les accidents avec des personnes blessées ont chuté respectivement de 20 et de $23 \%$, tandis que les réductions respectives pour les accidents avec blessés et les accidents mortels s'établissent à -20 et $-90 \%$ $\left(\right.$ ARRB, 2005) ${ }^{7}$. Enfin, à Victoria, le nombre d'accidents mortels aurait diminué de plus de $40 \%$ à la suite de la mise en œuvre du programme de contrôle (Cameron et al., 2003b).

Cependant, l'obtention de résultats satisfaisants ne doit pas masquer certaines limites liées à ce type de dispositif. En effet, les différentes études montrent l'existence d'un effet de halo spatial et temporel. L'effet dissuasif se révèle parfois éphémère. Concernant la dimension spatiale, l'effet de dissuasion se limite à environ deux kilomètres. Ainsi, la dissuasion produite s'avère passagère: les conducteurs adaptant leur vitesse de circulation après le passage au lieu de contrôle. Par ailleurs, le maintien des gains liés à la dissuasion exige la mise en œuvre permanente d'un système de contrôle. En effet, les conducteurs adoptent des stratégies de contrôle visant à contourner les dispositifs. L'étude de l'ARRB sur la Nouvelle-Galles du Sud souligne que l'extension du champ d'évaluation conduit à relativiser les résultats obtenus. Seule la réduction du nombre d'accidents mortels $(-57 \%)$ reste significative lorsque le périmètre d'investigation est élargi à l'axe de circulation. Une autre étude menée sur le Queensland met en évidence l'apparition possible d'effets de migrations d'accidents ${ }^{8}$. L'impact attribuable au CAV doit être dès lors relativisé (Champness et al., 2003: 149). L'effet de migration des accidents semble d'ailleurs être corroboré par l'étude de Newstead et Cameron (2003) qui met en évidence une augmentation de $18 \%$ du nombre d'accidents mortels entre 4 et $6 \mathrm{~km}$ après le lieu de contrôle. Un phénomène similaire peut être constaté pour les accidents avec blessés légers.

Quand bien même l'introduction d'un CAV permettrait l'obtention de résultats remarquables, il serait erroné de penser que disposer d'un tel programme suffirait à résoudre les enjeux de l'insécurité routière. Et cela, pour au moins deux raisons. Premièrement, l'implantation d'un CAV s'inscrit dans une politique et une stratégie de sécurité routière plus ambitieuse dans ses modalités d'action. Elle ne se limite pas à la

7. Évaluations effectuées aux sites de contrôle.

8. Les lieux d'accidents se déplacent vers une autre partie du réseau non contrôlée. En cela, le choix du périmètre d'investigation importe dans la mesure où il permet ou non d'inclure de tels effets de déport. 
seule question de la vitesse et à son volet répressif. L'introduction du contrôle aléatoire de l'alcoolémie, le port obligatoire de la ceinture de sécurité, la réduction des limitations de vitesse à $50 \mathrm{~km} / \mathrm{h}$ en agglomération participent à un effort général de réduction de l'insécurité routière en Australie. Le progrès technique contribue à l'amélioration de la sécurité des véhicules, tandis que la conception de l'infrastructure combine les exigences liées à la mobilité et les enjeux de sécurité (résorption des points noirs, entretien et conception du réseau routier, etc.). Ces différentes interventions interagissent et se renforcent mutuellement de telle manière qu'il devient parfois difficile d'attribuer précisément les effets à une seule composante du dispositif de sécurité routière.

Deuxièmement, la vitesse constitue un facteur important, mais qui n'explique qu'une partie de l'accidentalité. L'accident résulte en général d'une combinaison de différentes causes. L'inattention au volant, la fatigue, la consommation de substances illicites, des choix de conduite inappropriés et des problèmes de perception représentent autant de causes d'accident dont la vitesse peut aggraver les conséquences. Et pourtant, un CAV dispose de peu de prises sur de tels facteurs. L'intervention des autorités doit alors doser au mieux leur politique de sécurité routière et définir précisément les modalités pratiques de son action (ATSB, 2004: 30-31).

Toutefois, on retiendra des systèmes de CAV qu'ils permettent de réduire efficacement les vitesses de circulation les plus excessives, conduisant à influencer positivement le niveau de l'insécurité routière. Les ratios avantages-coûts des dispositifs se révèlent particulièrement élevés comme en NGS $(3,4)$ et spécialement au Queensland (supérieur à 40). Toutefois, ces modalités de contrôles doivent composer avec des limites liées aux stratégies de contournement de la part des conducteurs et les phénomènes de migration des accidents.

\section{Des enseignements stratégiques, organisationnels et institutionnels}

Les différents dispositifs opérationnels en Australie permettent de définir des enseignements stratégiques précieux pour les autorités désireuses d'implanter des CAV. Ces dispositifs s'appuient toutefois sur une gamme différente d'architectures organisationnelles et institutionnelles, soulignant à nouveau la richesse des expériences australiennes. 


\section{Les enseignements stratégiques}

Objectifs et principes stratégiques

Mettre en avant seulement l'effet dissuasif des CAV constitue une conclusion trop générale. En effet, l'implantation de tels dispositifs peut répondre au moins à trois autres objectifs: produire de la dissuasion générale, rechercher un effet de dissuasion spécifique et résoudre des problèmes localisés. La dissuasion générale repose sur l'hypothèse que la politique menée permet de dissuader les conducteurs de commettre une infraction à la limite de vitesse. Celle-ci doit être clairement distinguée de la dissuasion spécifique, qui consiste à sanctionner le contrevenant afin d'empêcher la récidive. On doit éviter ici de confondre surveillance et dissuasion générale, et ne pas assimiler la sanction à la dissuasion spécifique. En effet, la condamnation d'un contrevenant n'est possible que parce qu'en amont de la procédure un appareil de détection a pu l'identifier. De même, l'arrestation d'un contrevenant particulier peut produire une dissuasion pour les autres conducteurs. En fait, la ligne de démarcation entre les deux modalités de dissuasion repose sur la perception individuelle du processus. La distinction ne se fait pas à partir de caractéristiques objectives de l'outil d'intervention, mais à partir de la réception de ses effets par le conducteur (dimension subjective). L'objectif de résolution d'un problème localisé consiste à modifier durablement en un lieu précis la décision du conducteur, du fait, par exemple, de la dangerosité d'une infrastructure (tunnels, sortie d'école, portion d'autoroute dangereuse, etc.) ou d'une accumulation récurrente d'accidents (points noirs) (Lamm et Kloeckner, 1984).

Les différentes expériences menées en Australie permettent également d'énoncer des principes stratégiques précis (Cameron et al., 2003a). Un premier principe consiste à appuyer les dispositifs de contrôle par des campagnes publicitaires ciblées tant par leur contenu que par le médium utilisé (Cameron 1996). Il s'agit à la fois de jouer sur les anticipations des conducteurs qui établissent leurs choix de conduite en situation d'information imparfaite (Carnis, 2001) et de conférer une légitimité à la politique menée. L'intervention publique n'est pas menée à l'insu des conducteurs. Ses objectifs s'en trouvent également précisés: la politique ne peut plus reposer sur des décisions politiques isolées et arbitraires.

Les dispositifs mobiles banalisés produisent essentiellement une dissuasion spécifique. Les dispositifs fixes signalés doivent être utilisés 
pour résoudre des problèmes localisés: l'avertissement d'une menace doit inciter le conducteur à modifier ses choix de conduite en conséquence. Des dispositifs banalisés ou cachés peuvent produire également de la dissuasion générale à la condition qu'ils soient répartis sur l'ensemble du réseau. En fait, le conducteur dispose d'une information partielle: il ignore les modalités pratiques d'intervention du programme de contrôle mis en œuvre. Le dispositif joue ainsi sur cette incertitude pour fonder son pouvoir de dissuasion. Une implantation massive de dispositifs fixes peut produire également de la dissuasion générale, l'incertitude reposant ici sur la densité du dispositif et l'incapacité du conducteur à pouvoir intégrer le caractère massif des contrôles qui deviennent aléatoires. Enfin, la dissuasion du dispositif s'accroît avec l'introduction de dimensions aléatoires dans le fonctionnement des appareils: sites de contrôle fonctionnant de manière discontinue, zones de contrôle signalées mais pas les lieux eux-mêmes. Les modalités de fonctionnement du dispositif produisent elles-mêmes une situation d'incertitude pour le conducteur. Toutefois, il existe une dynamique d'apprentissage du conducteur qui progressivement cerne les dispositions stratégiques des autorités. Cet apprentissage plus ou moins rapide infléchit alors la portée dissuasive du dispositif. Oei a énoncé une belle formule pour désigner cette capacité d'adaptation: la théorie du saut du kangourou. Le conducteur réduit sa vitesse au lieu de contrôle pour l'adapter au niveau désiré après son passage (Oei, 1998). Les changements d'itinéraire obéissent également à cette logique. Il s'agit de contourner le dispositif. On pourrait ainsi généraliser la formulation de la théorie du saut du kangourou, en ne la limitant plus au lieu de contrôle, mais au dispositif lui-même.

\section{Complémentarité opérationnelle et approche économique}

Les expériences australiennes suggèrent un autre constat en soutenant que l'introduction d'un dispositif de contrôle automatisé de la vitesse ne s'accompagne pas de la disparition des activités traditionnelles de contrôle. En effet, chaque État a maintenu des activités traditionnelles de contrôle telles que les contrôles de vitesse avec interception (pistolets laser). L'introduction d'un CAV peut toutefois accélérer la modernisation des équipements de contrôle et conduire au remplacement des appareils existants (Harwood et al., 2001). En fait, il n'existe pas de choix monoproductif en matière de contrôle des vitesses. Les autorités couplent à 
la fois contrôles traditionnels et contrôles automatisés, ces derniers associant généralement dispositifs mobiles et fixes ${ }^{9}$.

Une approche économique du CAV permet également de préciser les champs du possible pour les autorités afin de mener une intervention efficace. En effet, il ne s'agit pas seulement de mettre en œuvre des dispositifs qui assurent des résultats positifs sur l'accidentalité, mais surtout de disposer de solutions idoines compte tenu des contraintes budgétaires $^{10}$. En cela, un CAV, quelle que soit sa forme, peut être interprété comme un coût fixe ${ }^{11}$ conséquent. Sa performance relative par rapport aux contrôles traditionnels ne devient significative que pour des flux de circulation relativement importants ${ }^{12}$. Les coûts fixes étant répartis sur un grand nombre de contrôles, le coût unitaire de contrôle diminue d'autant. En cela, l'avantage des CAV repose sur la possibilité de réaliser des économies d'échelle. Il s'ensuit logiquement qu'il est possible a priori de déterminer les zones d'intervention pour chaque type de dispositif. Les dispositifs automatisés fixes s'en trouvent particulièrement bien adaptés aux réseaux urbains à forte circulation. Les dispositifs automatisés mobiles disposent d'un avantage certain pour les axes interurbains et en rase campagne, tandis que les contrôles manuels peuvent être ciblés sur des axes à circulation de faible densité.

Un autre enseignement de l'approche économique repose sur la loi des rendements décroissants. Chaque niveau de surveillance supplémentaire conduit à une augmentation moins que proportionnelle des gains de sécurité. Autrement dit, les gains marginaux du dispositif diminuent. Empiriquement, cela s'en est trouvé confirmé à la fois au Queensland et à Victoria lors des renforcements des programmes de contrôle. Ainsi, le doublement des heures de contrôle à Victoria a conduit seulement à une réduction additionnelle des accidents corporels de $3 \%$. L'interprétation des résultats suggère qu'il existe une limite naturelle et une taille optimale des CAV. Les dispositifs de contrôle dissuadent en premier lieu les conducteurs les plus sensibles à la dissuasion. Progressivement ne subsistent que les plus réfractaires, c'est-à-dire ceux qui sont moins

9. Seuls les États très ruraux disposent en général uniquement de dispositifs mobiles.

10. On remarquera à ce titre que le MUARC (Monash University Accident Research Centre) a récemment remis un rapport au gouvernement d'Australie-Occidentale s'appuyant sur une telle démarche.

11. Le dispositif fixe s'avérant plus coûteux à l'unité, mais également en termes de coût d'opportunité. Les sites d'installation possibles sont d'emblée plus limités que ceux d'un dispositif mobile. 
disposés à modifier leurs choix de conduite. D'autres modalités d'intervention doivent être mises en œuvre pour les appréhender et les dissuader. Ce qui permet d'expliquer en partie l'existence de modalités complémentaires d'intervention.

\section{Les enseignements organisationnels et institutionnels}

La diversité des architectures organisationnelles et institutionnelles

Les différents programmes de CAV australiens mettent en évidence un éventail relativement varié d'architectures organisationnelles et institutionnelles que l'on peut désigner par des modèles de gouvernance, comprenant à la fois les agencements intra-organisationnels pour la prise en charge du contrôle du CAV et des relations spécifiques interorganisationnelles.

On peut distinguer tout d'abord différents modèles de gestion opérationnelle ${ }^{13}$ des CAV. La typologie proposée repose sur cinq modèles distincts. Le modèle policier se définit par une prise en charge opérationnelle complète du CAV par l'agence policière. Le Queensland Police Service maîtrise ainsi l'ensemble de la chaîne opérationnelle, faisant intervenir conjointement du personnel policier et civil (appartenant à son organisation). Le modèle administratif se caractérise par une prise en charge par des organisations gouvernementales non policières. Ainsi, l'Australie du Sud avait adopté ce modèle à la fin des années 1990. Le modèle privé consiste en la délégation à une entreprise privée de l'ensemble des tâches opérationnelles. Victoria a ainsi choisi à la fin des années 1990 la compagnie Tenix pour effectuer la gestion opérationnelle du dispositif ${ }^{14}$ mobile dans le cadre d'une contractualisation. Enfin, le modèle de séparation repose sur l'intervention de plusieurs organisations dans la gestion opérationnelle. En Nouvelle-Galles du Sud, le service de police gère les dispositifs mobiles, tandis que les dispositifs fixes

12. Cet avantage relatif des CAV est renforcé par l'incapacité technique et humaine des contrôles traditionnels à pouvoir traiter des flux importants.

13. Les modalités opérationnelles comprennent à la fois les opérations de contrôle, le traitement des informations, la gestion des poursuites et la gestion des données statistiques.

14. La police de Victoria dispose de prérogatives en matière de surveillance du bon fonctionnement du dispositif, c'est-à-dire des tâches qui pourraient être qualifiées de stratégiques. 
relèvent de la Roads and Traffic Authority, une organisation gouvernementale $^{15}$. Les autres États australiens s'articulent autour de modèles mixtes. Les tâches opérationnelles font l'objet de partage entre les services de police et des administrations, voire des entreprises privées (cas de la Tasmanie et des Territoires du Nord).

À ce stade de l'analyse, il n'est pas possible de déterminer la supériorité d'un modèle sur un autre. Néanmoins, quelques éléments de réflexion peuvent être avancés sur les avantages et les inconvénients associés à chaque modèle. Le modèle policier présente l'avantage d'une meilleure coordination entre les activités automatisées et traditionnelles du contrôle de la vitesse, mais il nécessite un protocole précis d'usage du dispositif afin d'éviter des phénomènes de substitution inopportuns entre les deux activités. Ce modèle comporte aussi le risque de présenter l'intervention policière uniquement sous un jour répressif et de générer une opposition systématique de la population à l'égard de son intervention. Le modèle administratif présente l'avantage d'éviter une concentration excessive du pouvoir de la gestion des vitesses au sein d'une seule organisation, mais nécessite en contrepartie des efforts de coordination entre les différentes agences. Des problèmes de rivalité peuvent également surgir entre les différentes organisations chargées du contrôle de la vitesse pour définir la stratégie appropriée. Le modèle privé offre la possibilité d'une grande réactivité et flexibilité dans l'usage des ressources, ainsi qu'une meilleure qualité de service dans la gestion du processus de contrôle. Il nécessite cependant une étroite coordination avec les autorités publiques et la mise en place d'une tutelle policière pour contrôler la légalité de la procédure. Des problèmes de crédibilité peuvent également apparaître. Un protocole précis et transparent doit alors être mis en place afin d'éviter les critiques traditionnelles d'enrichissement sur le dos des conducteurs. Le modèle de séparation semble être adapté lorsque des dispositifs spécifiques sont déployés à des enjeux précis (gestion de la sécurité des ponts et des tunnels, par exemple), mais exige en contrepartie des efforts de coordination entre les différentes organisations qui interviennent au sein du processus.

En conséquence, il n'existe pas un modèle de CAV qui se révélerait supérieur aux autres. Au contraire, la grande diversité des modèles met

15. L'analyse de l'information est sous-traitée à une firme privée. En cela, on pourrait également parler de modèles mixtes. 
en évidence la nécessité d'une certaine adaptabilité du dispositif aux contraintes institutionnelles qui différent d'un État à un autre. Cette typologie rudimentaire permet toutefois de souligner les différentes structures institutionnelles à l'œuvre, résultant sans doute de particularités historiques et organisationnelles propres à chaque État.

\section{Des pratiques convergentes}

Cette diversité institutionnelle ne doit pas toutefois masquer un agencement interorganisationnel récurrent pour chaque État, caractérisé par une imbrication des différents partenaires en ce qui concerne la dimension stratégique. Cette logique réticulaire introduit une réelle interdépendance entre les parties prenantes ${ }^{16}$, qui fait de la gestion du CAV un domaine partagé. Ces relations d'interdépendance exigent un large degré de consensus ${ }^{17}$ pour la mise en œuvre de ce type de dispositif, ce qui lui confère également une certaine assise institutionnelle, le rendant moins fragile et friable aux attaques dont il peut faire l'objet ${ }^{18}$. En reprenant le concept avancé par Anderson des « $4 \mathrm{C}$ » (contrôle, coopération, communication, continuité) (Anderson, 2000) ${ }^{19}$, on peut aisément démontrer des dimensions communes à l'ensemble des CAV australiens, fondées sur un contrôle indépendant des activités, la coopération interinstitutionnelle, la communication et l'amélioration du dispositif.

La politique des « $4 \mathrm{C}$ » s'appuie sur des mesures de contrôle diverses et agencées à différents niveaux. Ainsi, les forces de police, comme les

16. Nous avions mis en avant cette particularité pour la prise en charge des missions de police routière par le New South Wales Police Service (Carnis, 2005).

17. La recherche du consensus apparaît également clairement comme un élément de succès pour le cas du Québec. Les travaux de Paquette et Beaulieu (1996) ont souligné l'absence de consensus sur le radar photographique au milieu des années 1990, ce qui explique en partie l'impossibilité d'implanter un tel dispositif au cours de la décennie. Depuis un lent travail de maturation a été entrepris, ce qui a conduit entre autres à la remise du premier rapport de la Table québécoise de sécurité routière (2007), lequel préconise par sa quatrième recommandation la mise en place d'un projet pilote sur le radar photographique. La Table québécoise de sécurité routière a consisté à produire en partie le consensus nécessaire entre les différents acteurs clés pour rendre acceptable un tel projet. Cette expérience souligne le caractère crucial de cette dimension. Le projet pilot de radars photographiques a été repris par le ministre des Transports du Québec sous la forme d'une action prioritaire.

18. Ainsi en Ontario, le contrôle automatisé de la vitesse a fait l'objet d'enjeux politiques et électoraux qui ont conduit à son abandon.

19. En fait, on ne suit que partiellement Anderson et Edgar (2001) en redéfinissant quelque peu les catégories. En effet, Anderson proposait ce modèle pour expliquer le fonctionnement du dispositif fonctionnant sur Canberra. 
programmes de CAV font l'objet d'audits de la part d'organisations indépendantes privées et publiques. Le rapport remis par l'Auditor General de Victoria concernant le dispositif de CAV mis en ouvre dans cet État s'avère très illustratif à cet égard. Le contrôle repose sur l'atteinte des objectifs définis, la mobilisation de ressources idoines et la mise en œuvre correcte des moyens déployés (Auditor General Victoria, 2006). Une autre forme de contrôle consiste en l'évaluation récurrente des dispositifs de contrôle (Cameron et al., 2003; Edgar, 2001). À ce titre, les experts et les centres universitaires spécialisés (MUARC à Melbourne, CARRS- $\mathrm{Q}^{20} \mathrm{du}$ Queensland) produisent régulièrement des études, dont les résultats sont publicisés auprès des pairs, des praticiens et des policiers lors de conférences annuelles (ARRB, Road Safety Research, Policing and Education Conference). Sans vouloir être exhaustif sur la dimension du contrôle, il convient également de souligner l'utilisation des rapports annuels des services de police comme moyen de mise en perspective des résultats obtenus et leur utilisation pour corriger éventuellement les stratégies poursuivies par un système élaboré de collecte d'informations et de cartographie.

Une autre dimension essentielle de ces dispositifs repose sur la coopération étroite entre les différentes parties. Les services de police collaborent avec les services techniques municipaux et centraux du ministère des Transports, auxquels se joignent parfois des organisations d'usagers de la route, les assureurs publics en charge de l'indemnisation des préjudices corporels, mais aussi le ministère de la Justice. Les usagers peuvent être associés pour valider le choix des lieux de contrôle, tandis que les assureurs publics voient dans ces dispositifs un moyen de réduire la charge financière liée aux accidents de la route. D'ailleurs, les assureurs publics s'associent régulièrement aux services de police en finançant des opérations de contrôle supplémentaires; cette dépense représente une forme d'investissement dont le retour doit se traduire par une réduction des indemnisations versées à la suite de la diminution du nombre d'accidents corporels. La coopération entre les partenaires se traduit également par une réelle proximité avec les milieux universitaires fournissant expertise et analyse, et qui voient en retour l'accès facilité au terrain et aux informations, tant par les services de police, que par les assureurs publics et les différents ministères. La convergence 
des intérêts des différentes parties permet ainsi une intégration des actions des partenaires et l'échange de vues sur ce qui est mis en œuvre ou pourrait être fait.

La communication constitue une dimension essentielle du fonctionnement des dispositifs (Cameron, 1996). On a mis l'accent sur cette caractéristique tant d'un point de vue dissuasif que celui de la stratégie de la légitimation qu'elle peut produire. La communication s'appuie à la fois sur des campagnes publicitaires plus ou moins intenses, des informations diffusées par l'intermédiaire des différents médias sur la mise en œuvre du programme de contrôle, mais aussi sur les efforts consentis par les autorités en termes de signalisation des lieux ou des zones de contrôle pour avertir les usagers. Les efforts de communication reposent également sur la diffusion et l'accessibilité des informations, des études réalisées par les experts, des rapports d'audit et d'évaluation, et par leur mise à disposition auprès du grand public. La communication résulte également de l'échange de bonnes pratiques au cours de conférences internationales entre experts, qui constituent un réel carrefour où se brassent les différentes pratiques et se confrontent systématiquement les résultats obtenus à la suite de la mise en œuvre de stratégies particulières.

La dimension de continuité suggère la mise en œuvre d'une politique de contrôle qui s'inscrit dans le temps. Elle implique en conséquence une temporalité, notamment par la mise en œuvre du dispositif avec des étapes successives de développement. Le cas précurseur de l'État de Victoria ou encore le renforcement considérable du programme de contrôle au Queensland illustrent ce déploiement par phases. La continuité du programme implique également la mise en œuvre d'efforts pour améliorer le dispositif (audits menés, politique systématique de comparaison entre États, mesures d'évaluation, etc.). Enfin, la durabilité de tels dispositifs nécessite de penser leur acceptabilité et de définir, entre autres, les moyens de favoriser la coopération interinstitutionnelle. Cela exige également de réfléchir à un travail de légitimation des dispositifs. Ainsi, apparait une réelle interdépendance entre les différentes dimensions mentionnées: chacune est difficilement compréhensible sans la prise en compte des autres. 


\section{Conclusion}

Les systèmes de contrôle automatisé de la vitesse australiens constituent un éventail intéressant d'interventions possibles. Ils représentent un outil relativement performant pour diminuer de manière significative les vitesses de circulation et l'accidentalité. Les différentes manières de procéder reflètent sans doute les particularités géographiques et institutionnelles propres à chaque État, et ce, malgré leur proximité géographique. Elles soulignent aussi une certaine flexibilité dans la mise en œuvre d'un tel dispositif et sa capacité d'épouser les spécificités de l'environnement dans lequel il est inséré.

Ce qui reste remarquable est la tension existante entre à la fois la diversité des dispositifs et la convergence des enseignements, comme si la différenciation était maîtrisée. Ainsi, les pratiques sont diverses, les stratégies recherchées et les mises en œuvre différenciées, et pourtant les résultats en termes de réduction de l'insécurité routière convergent, tandis que des enseignements opérationnels peuvent être dégagés. Alors que les architectures organisationnelles et institutionnelles se distinguent parfois de manière radicale, les systèmes de CAV reposent étonnamment sur des principes de mise en œuvre opérationnelle similaires (les $4 \mathrm{C}$ ).

Les expériences australiennes constituent ainsi un réservoir d'enseignements fort appréciable pour des autorités qui désirent mettre en œuvre un tel dispositif de contrôle. Les négliger serait préjudiciable. La facilité avec laquelle ces dispositifs s'adaptent aux spécificités locales fait que les résultats obtenus avec ces expériences peuvent être réutilisés aisément par d'autres structures institutionnelles.

\section{Références}

Anderson, R., \& Edgar, A. W. (2001). Mobile Speed Cameras in the ACT Slashing Speeds and Cutting Crashes. In Proceedings of Road Safety Research, Policing and Education Conference. Melbourne, Australia.

ARRB (Australian Road Research Board) (2005). Evaluation of the Fixed Digital Speed Camera Program in NSW, Report for Roads and Traffic Autbority. NSW, RC 2146, May. Victoria: ARRB Transport Research Ltd.

ATSB (Australian Transport Safety Bureau) (2004). Road Safety in Australia, A Publication Commemorating World Health Day. Canberra, ACT: Australian Government.

Auditor General Victoria (2006). Making Travel Safer: Victoria's Speed Enforcement Program. Melbourne: Victoria Government Printer. 
Bourne, M. G., \& Cooke, R. C. (1993). Victoria's Speed Camera Program. In R. V. Clarks (ed.), Crime Prevention Studies (1, 177-192). New York: Criminal Justice Press.

Cameron, M. (1996). Mass Media Publicity Supporting Police Enforcement and its Economic Value. Proceedings of the Symposium on Mass Media Campaign in Road Safety (29-58). Scarborough Beach, Western Australia.

- (2005). Automatic Enforcement of Vehicle Speeds. Presentation at INRETSGARIG. 27 octobre 2005, miméo. Paris, France.

Cameron, M., Delaney, A., Diamantopoulou K., \& Lough, B. (2003a). Scientific Basis for the Strategic Directions of the Safety Camera Program in Victoria. Report $\mathrm{n}^{\circ}$ 202, Victoria: Monash University Accident Research Center.

Cameron, M., Newstead, S. V., Diamantopoulou, K., \& Oxley, P. (2003b). The Interaction Between Speed Camera Enforcement and Speed-Related Mass Media Publicity in Victoria, Australia. 47 $7^{\text {th }}$ Annual Proceedings Association for the Advancement of Automotive Medicine, 22-24 September 2003, Melbourne, Victoria.

Carnis, L. (2001a). Entre intervention publique et initiative privée: une analyse économique en sécurité routière. Une application aux législations sur la vitesse. Thèse de doctorat de Sciences économiques, Faculté des Sciences économiques et de Gestion, Université de Reims Champagne-Ardenne.

- (2001b). L'apport de l'analyse économique au contrôle des excès de vitesse. Revue Politiques et Management Public, 19 (2), 83-103.

- (2005). Les missions de police routière du New South Wales Police Service, une spécialisation en danger? Les Cabiers de la Sécurité, Revue Trimestrielle de Sciences Sociales, 58, 75-99.

Champness, P., \& Folkman, L. M. (2003). The Impact of Speed Cameras on Speed-related Crashes over Time. Proceedings Road Safety Research, Policing and Education Conference, (2, 149-154). Sydney, New South Wales, Australia.

Champness, P., Sheehan, M., \& Folkman, L. M. (2005). Time and Distance Halo Effects of an Overtly Deployed Mobile Speed Camera. In Proceedings Road Safety Research, Policing and Education Conference, Wellington, New Zealand.

Edgar, A. (2001). Evaluation of the Effectiveness of Speed Cameras in the ACT. NRMA-ACT Road Safety Trust, Final Report. Victoria, Australia: ARRB Transport Research Ltd.

Harwood, C., McInerney, R., Cairney, P., Toomath, J., Evans, J., \& Swadling, D. (2001). Speed Enforcement in Australasia. Sydney, Australia: Austroads Incorporated.

Lamm, R., \& Kloeckner, J. H. (1984). Increase of Traffic Safety by Surveillance of Speed Limits with Automatic Radar Devices on a Dangerous Section of a German Autobahn: A Long-Term Investigation. Transportation Research Record, 974, 8-16.

Newstead, S., \& Cameron, M. (2003). Evaluation of the Crash Effects of the Queensland Speed Camera Program. Report n ${ }^{\circ}$ 204. Clayton, Victoria: Monash University Accident Research Center. 
Oei, H-L. (1998). The Effect of Enforcement on Speed Behaviour: a Literature Study. Part of the MASTER Project funded by the European Commission. D-988 - SWOV Leidschendam, june.

Opp, D. O., \& Sebold, F. D. (1972). Quasi Returns-To-Scale in the Provision of Police Services. Public Finance, 27, 46-61.

Paquette, G., \& Beaulieu, N. (1996). Efficacité et pertinence des radars photographiques dans le contrôle des vitesses. In Santé urbaine par les transports (2, 212-228). Québec: Université Laval, ATQR.

Table québécoise de la sécurité routière (2007). Pour améliorer le bilan routier, Premier rapport de recommandations de la Table québécoise de la sécurité routière.

\begin{abstract}
The automatic speed enforcement (ASE) constitutes an innovative public intervention to reduce the number of road victims. This particular technique of speed enforcement makes possible a more intensive control of driving speeds, the increase of the potential punishment for speed offenders and an acceleration of the prosecution process. Such a program was implemented initially in Victoria, Australia during the 80 's. At present, similar speed enforcement programs have been operated throughout the different Australian States. They present real differences and were designed to reach special objectives and strategies of traffic safety. Nevertheless a common conclusion can be drawn: these programs constitute really efficient means to reduce the number of road victims. The article stresses the existence of different organizational and institutional designs for operating such programs and consequently a particular easiness for implementing them in specific institutional environment. However all those systems rest upon important principles, the " $4 \mathrm{C}$ " (Control, Co-operation, Communication and Continuation), implying strong relationships between the key players.
\end{abstract}

RESUMEN El control automatizado de la velocidad (CAV) es un medio novedoso de intervención de las autoridades para reducir el número de víctimas de la circulación vehicular. Esta técnica de detección hace posible un mayor control de la velocidad, aumenta el potencial de sanción de los infractores y agiliza los procedimientos judiciales. Este tipo de dispositivo se introdujo por primera vez en Australia en el estado de Victoria a mediados del decenio de los ochenta y desde entonces se ha generalizado a las demás entidades. Los dispositivos utilizados son muy variados y se apoyan en diversas estrategias, aunque todas se orientan al mismo resultado: la disminución significativa de los accidentes de circulación y del número de víctimas. Resultan, pues, eficaces. Por otra parte, dichos programas de control se apoyan en varias arquitecturas organizacionales e institucionales, lo que les confiere cierta flexibilidad en cuanto a su implantación en distintos medios. Todos los sistemas se apoyan, sin embargo, en los principios simples de las " $4 C$ " (control, cooperación, comunicación y continuidad), que requieren la integración de diferentes actores clave. 